\title{
A system for monitoring real-time body parameters of sows using a lightweight and flexible wireless sensor platform
}

\author{
M. Kjeldsen ${ }^{1}$, T. Gregersen ${ }^{2}$, S. Wagner ${ }^{3}$, C.G.V. Bøgh ${ }^{4}$, C. Nielsen ${ }^{5}$
}

\section{N F O}

Received 20 Oct. 2015

Accepted 23 Mar. 2016

Available on-line 30 Apr. 2016

Responsible Editor: M. Herdon

\section{Keywords:}

Sow behaviour, wireless sensor network, ear tag, flexibility.

\begin{abstract}
A B S T R A C T
Temperature and head movement are relevant parameters when analyzing the farrowing behavior of sows. Obtaining these body parameters in a way that is nonintrusive to animals is a major challenge in the harsh farrowing pen environment. Due to the presence of large amounts of metal as well as the unpredictable behavior of animals, such environments are not ideal for wired communication platforms. Intrusive measuring equipment may cause animals to deviate from their normal behavioral patterns invalidating gathered sensor data. Using lightweight, highly mobile and wireless sensor equipment is thus essential for unobtrusive measurements. This article discusses the challenges involved in developing a lightweight and flexible wireless sensor network infrastructure platform used in the analysis of sow behavior during farrowing. The platform is based on the customizable wireless sensor platform Shimmer and the open source software frameworks TinyOS and SPINE. Embedded in hot melt adhesive, the Shimmer modules were used as ear tags providing biologists with head movement and temperature data throughout six months. Focus is on the technical aspects of developing a system faced with mutually exclusive and changing requirements in an iterative and progressive research project, drawing upon the experiences from several stages of live experiments with farrowing sows.
\end{abstract}

\section{Introduction}

Obtaining continuous body parameters for classifying animal behavior is useful for automating and improving critical agricultural processes (Moreau et al. 2009), (Roelofs et al. 2005), and (Cornou \& Lundbye-Christensen 2012). One such process, the farrowing of sows, is often associated with a high piglet mortality rate (up to $22.4 \%$ ) as discussed by Oliverio et al. (2008) and Fernandes et al. (2011). Reducing piglet mortality requires timely manual assistance from farm staff members. This is not always feasible in modern farming as many processes have been automated and human presence in the farrowing pen during critical situations cannot always be relied upon.

The need for measuring body parameters on animals has been investigated for more than a decade. Early research by Frost et al. (1997) indicates a need for measuring body temperature and heart rate. Wathes et al. (2008) describes the need for an integrated system that manages interlinked biological and physical processes of livestock production while Cornouand \& Lundbye-Christensen in series of studies $(2008,2010,2012)$ has investigated the behavior of farrowing sows. Different approaches for measuring body parameters on animals have been investigated. Ulrich et al. (2008) describes the ALTpedometer (Activity, Lying time and Temperature) which detects oestrus duration and oestrus intensity for dairy cattle. The detection of activity is achieved by using piezo-accelerometers and inactivity by using photo sensors through a device mounted at one of the forelegs. Guo et al (2009) describes how an accelerometer, a GPS-receiver, and a radio transceiver were mounted in a neck collar to observe the behavior of cows. They were able to perform sleeping, grazing, and ruminating behavioral analysis for groups of cows based on position tracking and acceleration. Cornou \& Lundbye-Christensen (2008) used a neck collar based monitoring system to observe the behavior of

\footnotetext{
${ }^{1}$ Martin Kjeldsen, Mjølner Informatics A/S, Finlandsgade 10, 8200 Aarhus N, Denmark

2 Torben Gregersen, Aarhus University School of Engineering, Finlandsgade 22, 8200 Aarhus N, Denmark

${ }^{3}$ Stefan Wagner, Department of Engineering, Aarhus University, Finlandsgade 22, 8200 Aarhus N, Denmark

${ }^{4}$ Christian G. V. Bøgh, Skov A/S, Hedelund 4, Glyngøre, 7870 Roslev, Danmark

${ }^{5}$ Carsten Nielsen, Landmeco A/S, Haulundvej 16, 6870 Ølgod, Danmark
} 
pigs in an indoor stable environment by using accelerometer and Bluetooth radio communication. They describe how the sows' neck collars tended to loosen after few days and further discuss whether accelerometers should be placed on ear tags instead (Cornou \& Lundbye-Christensen 2010).

When observing body parameters of animals, it is important for the applied technology to remain nonintrusive. It is impossible to predict animal behavior when introducing foreign objects into a habitat, and any interaction with the environment can potentially invalidate data. Transferring data via physical cables may prevent animals from moving freely, contaminating data through interaction. Wireless communication, though presenting other challenges (such as battery life time), allow us to observe more discretely. An in depth discussion of the challenges of such a wireless monitoring system is discussed in section 2 .

Previous approaches to monitoring systems have thus relied on mounting measuring equipment in places that allows for the weight of the equipment to be disregarded. As a consequence, sensor data quality may be affected out of consideration for animal welfare and practical concerns. In this paper, we present a case study in which a lightweight wireless sensor network is used for continuous monitoring of head-movement and body- and surrounding temperature for a group of farrowing sows while keeping the technology as nonintrusive as possible. Requirements to sensors, population size, habitat, and data gathering, was planned to change progressively during the project lifecycle.

The aim of this study was to determine whether it is feasible to construct a lightweight flexible wireless sensor infrastructure platform that is: easy to reconfigure and deploy, able to support an iterative and progressive research agenda, while also being sufficiently robust for real world deployment in the harsh environment of a pig pen.

\section{Challenges}

In order to gather biological data on sow behavior in the farrowing pen, a sensor platform was needed that would facilitate several iterative substudies to evaluate the feasibility of using ear-mounted accelerometers, temperature sensors, and possibly other sensor types, for the individual animal and surroundings, in order to track and identify pig farrowing behaviour. Also, the sensor platform needed to be wireless and should be able to measure farrowing behaviour for the entire duration of the farrowing process.

As the platform was intended to evolve based on results from progressive experiments at AU Foulum $^{6}$, where non-technical research staff should be able to (re)use and (re)configure the equipment in several substudies, the platform needed to be highly flexible and configurable. Furthermore, the sensor platform should be mature enough to evolve into a final product.

The first prototype was required to support obtaining accelerometer data from a single sow for initial data analysis purposes. The second prototype was required to support up to six sows simultaneously using two additional temperature sensors. Later experiments required additional pens and animals to be included. Important design criteria were: to support changing accelerometer usage and configuration as well as to design and implement a communication model with only minimum changes to hardware and software design needed when changing scope and focus of the individual animal studies.

\footnotetext{
${ }^{6} \mathrm{AU}$ Foulum is part of Aarhus University and is home to most of the university's research in food and agriculture. Research areas include plants, animals, food, organic farming, bioenergy, environment, climate, soil, genetics and technology, including pig behaviour studies, as was the case for this project. AU Foulum has flexible housing facilities for experiments with pigs, cattle, poultry and horses and a mink farm. The facilities include an experimental slaughterhouse, a feed mill and an intensive care unit. The intensive care unit contains a wide range of facilities, including highly specialised operating theatres, an infection unit, a rat house and isolators. 
Several challenges were identified, arising from conflicting requirements:

1. The ear tag mounted sensor devices should be able to transmit data continuously in a reliable way for a long period, at least three weeks, gathering data before, during and after the farrowing process. Continuous transmission of sensor data leads to high power consumption requiring heavier battery packs.

2. Data measured by the sensors on the ear tag should be as accurate as possible and should be sent continuously with time stamps for real-time monitoring, as well as asynchronous data analysis. Such sensor equipment tends to be inaccurate necessitating individual calibration of sensor platforms.

3. The weight of the ear tag should be as low as possible to avoid irritating the pigs' ears, i.e. not exceeding 65 grams if possible, in order not to be cumbersome for the pig to wear. This calls for a lightweight hardware design including limited battery size and capacity.

4. The ear tag should be encapsulated in a way that makes it resistant to dust, dirt, ammonium gasses, and the mechanical stress that occurs when a sow shakes her head or the ear tag collides with the metal bars in the farrow pen. Increased protection increases weight of the ear tag.

5. Wireless communication in farrowing pens can be problematic as these pens are often constructed with iron bars leading to reflection and attenuation of radio waves. Furthermore, the sow's body leads to an attenuation of the radio waves if she is lying on the radio antenna. Increased radio stability may lead to higher power usage.

6. Requirements to hardware functionality and configuration were expected to change due to the iterative nature of the project. The project was planned to start with initial basic data gathering and analysis before moving on to large scale data collection. Therefore, both hardware and software infrastructure needed to be flexible and open for reprogramming and sensor refitting as needed.

Thus, low weight and long operating lifetime were some of the mutually exclusive goals of the project. Therefore, a main challenge of the project was to discover the optimal combination of weight and operating life time with data transfer being as reliable as possible.

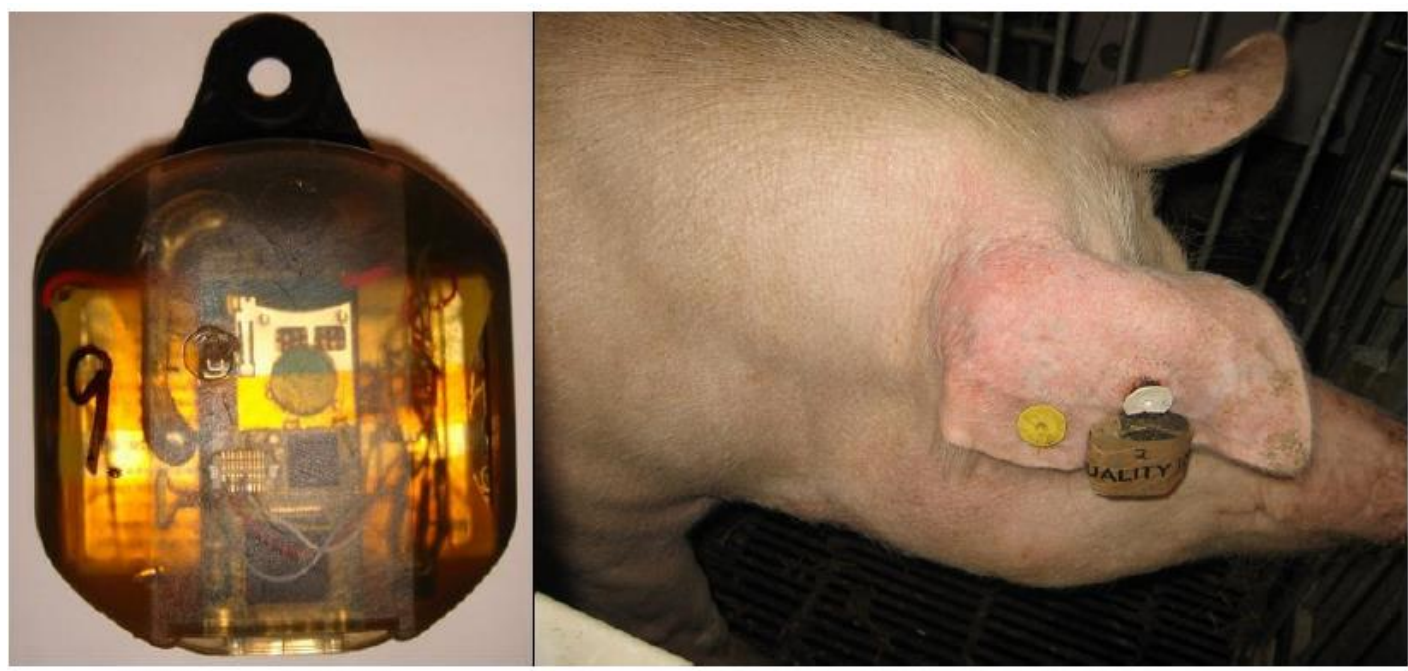

(a) Embedded in hot melt adhesive

(b) Mounted on farrowing sow

Figure 1. a) Ear tag embedded in hot melt adhesive, and b) placement on pig, mounted on the ear 


\section{Materials and methods}

For the ear tag to remain a nonintrusive entity in the farrowing pen environment, the most important attribute was its low weight. Secondly, a wireless sensor platform that allowed sows to move freely and unhindered was crucial to maintaining the validity of the sensor data. Operating lifetime based on battery size and low power operation weighed heavily as well. Finally, the need for a flexible and configurable platform was also a major requirement. Constructing a flexible and reliable infrastructure platform would either require building a dedicated hardware platform or selecting a well-tested, low power wireless sensor hardware platform. As we needed a high degree of flexibility in the project including progressive experimentation with different sensors (accelerometer and temperature) we decided to build on an existing standard hardware platform. Also, we considered using a platform with a low power operating system, in order to be able to deploy advanced software algorithms and communication models.

Shimmer, Micaz, and Telosb are examples of small form factor, low power, and wireless sensor platforms (Johnson et al. 2009) which were considered relevant to employ as sensor platforms. Table 1 shows a comparison between wireless sensor platforms of interest emphasizing some of the important criteria that were used to choose the sensor platform for the ear tag (Johnson et al. 2009).

Table 1. Mote comparison. Data from (Johnsen et al. 2009).

\begin{tabular}{|c|c|c|c|}
\hline & Shimmer & TelosB & Micaz \\
\hline Radio Chip & $\begin{array}{l}\text { TI CC2420, } \\
802.15 .4 \\
\text { compatible }\end{array}$ & $\begin{array}{l}\text { TI CC2420, } \\
802.15 .4 \\
\text { compatible }\end{array}$ & $\begin{array}{l}\text { TI CC2420, } \\
802.15 .4 \\
\text { compatible }\end{array}$ \\
\hline Onboard sensors & $\begin{array}{l}\text { Accelerometer, } \\
\text { Gyroscope }\end{array}$ & $\begin{array}{l}\text { Humidity, } \\
\text { temperature, } \\
\text { light }\end{array}$ & None \\
\hline TinyOS support & Yes & Yes & Yes \\
\hline Weight & $\begin{array}{l}10.36 \mathrm{~g} \text { (w. } \\
250 \mathrm{mAh} \text { Lith- } \\
\text { ion battery) }\end{array}$ & $\begin{array}{l}63.05 \mathrm{~g}(\mathrm{w} .2 \\
\text { AA batteries) }\end{array}$ & $\begin{array}{l}63.82 . \mathrm{g}(\mathrm{w} .2 \\
\text { AA batteries) }\end{array}$ \\
\hline
\end{tabular}

All platforms have on-board sensors supported natively by TinyOS allowing for faster development time and all support wireless radio transmission. The weight of both the TelosB and the Micaz, however, were more than the allowed weight of the final ear tag, and with a weight of 10.36 grams the Shimmer sensor platform was chosen. The Shimmer platform has been used successfully in related sensor network projects (Yang et al. 2009).

\subsection{Software Frameworks}

In order to facilitate a flexible development process, the system was based on existing software frameworks exclusively. This included TinyOS which is a component-based operating system for small, resource constrained wireless sensor networks (WSN). TinyOS is an open source operating system designed for wireless embedded sensor networks and features a component based architecture that enables fast development while minimizing code size which is often required by the memory constraints inherent in small form factor sensor networks. TinyOS's component library includes 
network protocols, distributed services, sensor drivers, and data acquisition tools all of which can be used as-is or refined for custom behaviour.

SPINE (Signal Processing In Node Environment) is a software framework for the design of Wireless Sensor Network applications. SPINE is developed as a TinyOS application and enables speedy development of systems that can be used for analysis and classification of sensor data through its built in signal processing libraries. A SPINE application is deployed on the sensor platform, and sensor data is handled at the server side through an API developed in the Java programming language. SPINE has been used in related wireless sensor network case studies (Johnson et al. 2009).

Combined, TinyOS and SPINE support features such as over-the-air configuration of multiple nodes and flexible integration of new drivers for custom sensor hardware extensions. Both frameworks offer drivers for basic sensor types like accelerometers, temperature sensors, and gyroscopes and support a heterogeneous and flexible environment.

\subsection{Encapsulation}

Ammonia, which can be found in all livestock stables, is a gas with alkaline properties and a corrosive effect that requires shielding of sensitive hardware to avoid malfunction. During the first pilot study of the project, Shimmer devices were encased in simple plastic containers and attached to the ears of the sows with crude tape, but they stopped working after a short amount of time due to the lack of proper shielding. In order to allow the Shimmer sensing hardware to survive in such a hostile stable environment, they were embedded in a hot melt adhesive during the final stages of the second ear tag prototype construction. A picture of the fully functional ear tag featuring the onboard accelerometer, a $1200 \mathrm{mAh}$ battery and two temperature sensors, can be seen in Figure 1. The transparent nature of the hot melt adhesive allows the LEDs on the Shimmer platform to remain visible for problem detection purposes. The Shimmer platform connector port used for programming and recharging had to be left accessible and was covered with tape during operation, increasing the size of the ear tag slightly.

\subsection{Sensor network configuration}

SPINE allows for network configurations to be changed dynamically through server-side, over-theair configuration of a network of sensors, which also allows configuration from remote locations. Due to the star topology nature of the sensor network, only the nodes immediately visible by the base station handling configuration requests could be configured.

Through a custom and textual configuration format, super users of the system could manipulate central configurations to change the workings of each ear tag in the sensor network such as sample rate and amount of samples contained in each transmission.

In order to avoid data loss and reduce data communication power usage, an extended local data cache on each ear tag was considered as a design alternative. Such a solution could only be used to transmit data wirelessly when signal strength conditions would be optimal, reducing lost data package levels and power use. This only includes transmitting data when being near a data collection unit allowing for low power communication. The Shimmer device features an SD-card storage facility. However, according to Shimmer specifications, the SD-card hardware is not designed for low power operation which was confirmed by initial testing efforts. Rather than implementing such a caching strategy, each transmission was optimized to contain as much sampled data from each sensor as possible according to an agreed upon sample rate that would optimize the amount of data gathered while allowing data to be monitored in real time. During initial tests of the ear tag, a sample rate providing the best balance between data coverage and battery-lifetime was determined in order to transmit sampled data as infrequently as possible, prolonging battery lifetime. Each transmission contained a series of samples from each sensor, represented as 12-bit values, utilizing the maximum possible amount of data in each message header. While a local cache and a deferred communication 
strategy might prove more energy efficient, this would require us to employ a dedicated hardware platform instead.

Due to the layout of the farrowing pens, each base station could cover only a limited number of ear tags. For the system to receive data from larger quantities of ear tags, more base station nodes had to be deployed. No effort was made to configure the signal range of base stations in order to avoid an intersection of ear tags and picking up sensor data from the same ear tag at multiple base stations. Figure 2 depicts a scenario where each sow is placed in a big farrowing pen. The radio waves have free passage due to the size of the pen. In this case two base stations were able to receive the measurements. The reception radius from two base stations overlaps. No effort was made to discard duplicate transmissions during run time. Instead, these entries were discarded during post processing.
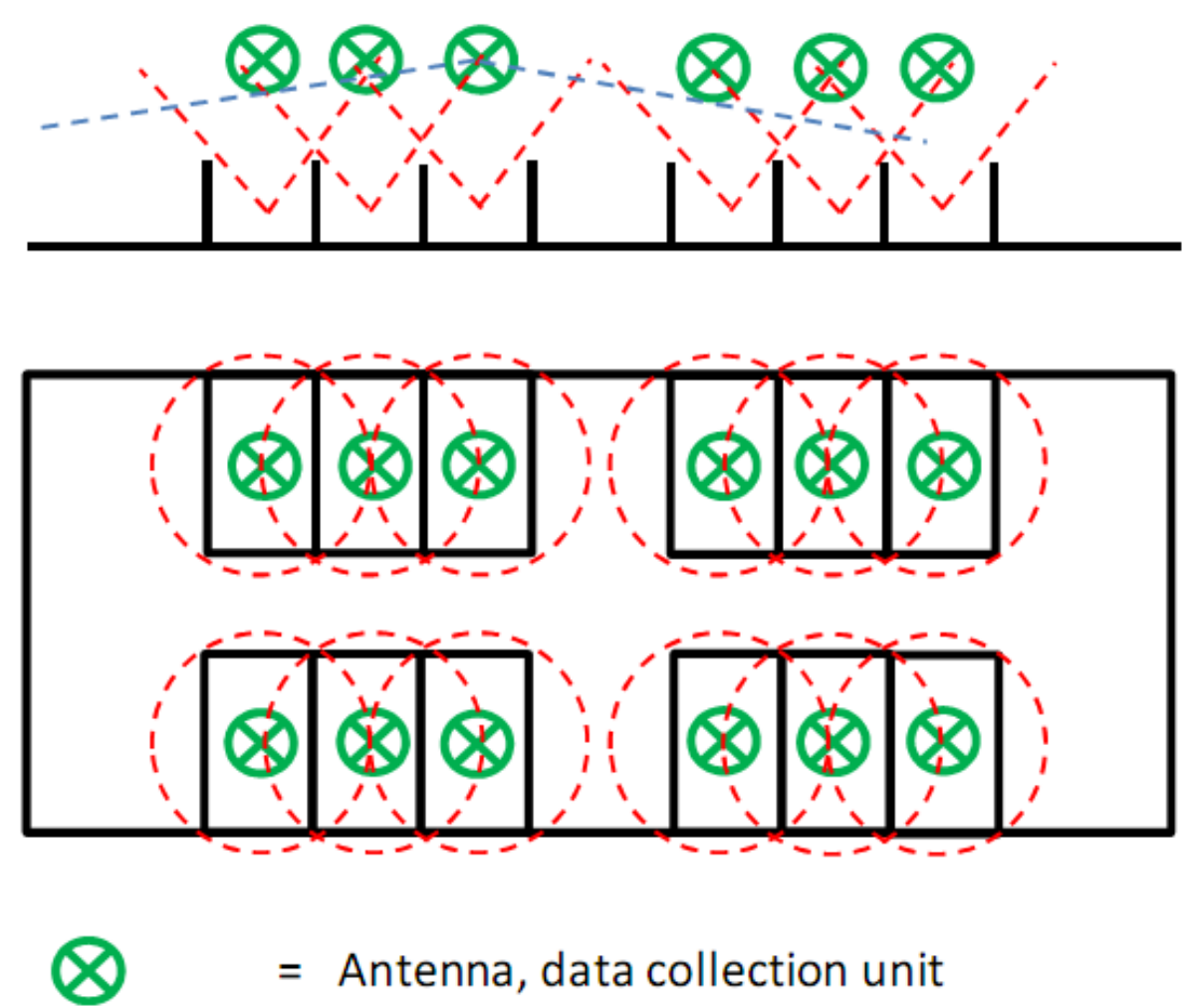

$=$ Antenna, data collection unit

$=$ Limit for each data collection unit's radio coverage

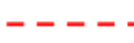

$=$ Angle limit for radiation from sow's ear tag

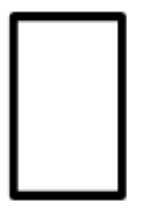

= Small pen

Figure 2. Deployment of sows placed in small pens, seen from the side and from above 


\subsection{Analysis and design}

Following standard systems engineering analysis methods, field visits were made to AU Foulum research center to inspect the farrowing pens in order to gather input for the technical requirements including communication technology, environment analysis, and physical constraints of the sows. Also, several meetings were held with both engineers and Foulum biologists researchers in order to establish functional requirements for each sub study with a focus on purpose and duration of the planned substudies as well as required sensor support and data export format.

\subsection{Evaluation}

After gathering system requirements and deciding on basing the infrastructure platform design on the Shimmer with TinyOS and SPINE, an initial version of the ear tag was produced. The main purpose of this pilot study was to collect data from a single sow, gathering accelerometer data only. This allowed the AU Foulum researchers to perform data analysis on the accelerometer measurements and deduce required changes to data gathering and communicating algorithms, sensor calibration, as well as identifying communication and battery limitations. The special purpose linearity device was constructed to facilitate the calibration process, and all sensor devices were calibrated.

In the next pilot study, six sows were monitored with the second generation ear tag which included the two temperature sensors. These ear tags were calibrated so their accelerometer data could be used for precise measurements. Initially, data from up to six ear tags was collected by one data collection unit to test the basic functionality. Data was collected on a dedicated computer running Windows, the SPINE base station framework, and our specialized test application.

Following this, groups of maximum twelve sows were equipped with ear tags in big farrowing pens. This required the addition of a second data collection unit in order to cover the entire pen area. The data collection units were placed with an overlap to ensure that no data would be lost.

Finally, the infrastructure platform needed to support twelve sows in small farrowing pens. It was necessary to place a data collection unit above each pen, most likely caused by the upward radio wave radiation from each pen.

\section{Results}

The constructed infrastructure platform was successfully used in 15 substudies of the farrowing sow research project at AU Foulum. The development team was able to gather the required type and amount of data in the various phases of the project, adapt the infrastructure platform with new sensors, change algorithms and calibration, as requested by the Foulum research team after the first two substudies. A total of 125 sows were equipped with ear tags containing a wireless sensor device; first up to 6 sows at a time and later up to 12 sows at a time, in small and large farrowing pens. In all, 53 sows were tested in small farrowing pens and 72 sows were tested in large pens. Each sow was measured during the three weeks they spent in the farrowing pen. The data was successfully used by biologists to analyse sow behaviour in farrow pens based on acceleration and temperature data. In total, the sensor network collected 32 Gigabytes of data represented by 385 million accelerometer and temperature measurements. Furthermore, during the test period only a few ear tags were lost, and the irritation of the sow's ears was negligible. Finally, the battery life time was sufficient to gather the required data.

\section{Discussion}

Based on the successful usage of the reported lightweight sensor platform in 15 substudies, we conclude that it is indeed feasible to construct a flexible wireless sensor infrastructure platform that is easily reconfigurable and deployable and able to support an iterative and progressive research agenda, as stated in the aim of the study. Also, we found that the platform was sufficiently robust for real world deployment in the harsh environment of a pig pen, thus fulfilling the main objectives of the 15 pig farrowing studies. Also, battery life time was sufficient to achieve the biologist's requirements of three weeks of uninterrupted data collection, despite the special environment a pig pen constitutes. 
We argue that there is a lack of relevant research platforms for studying pig farrowing behavior and related studies. Thus, although Marchioro et al. (2011) and Fernandes et al. (2011) both report on similar lightweight approaches based on dedicated hardware platforms, neither Marchioro et al. or Fernandes et al., nor any other studies reported in literature, provides a relevant lightweight platform solution similar to the one presented in this study; a platform that is able to support the flexible and iterative research agenda needed for this type of observational studies. This include our choice of sensor placement on the ear of the pig, rather than a collar as suggested by Cornou \& LundbyeChristensen (2008 \& 2010)

However, due to radio link disturbance caused by radio wave reflections some measurements were lost. This instability in the radio link also caused issues related to the configuration of the sensor network of tags. If one of several configuration messages from the base station to the ear tag was lost, that ear tag would have to be reset physically. This was, however, considered to be a minor fault that only applied to the configuration phase. It could be solved by manual inspection of data and sensor reset without obstructing the data gathering process.

Changes in multipath propagation of radio signals caused by natural movements of the sow sometimes compromised the link quality of the ear tags for brief periods. Radio link quality was also compromised, sometimes for longer periods, when a sow laid on her ear tag, because of the inability of radio frequencies to penetrate body fluids. Given the small batteries of the tags due to the low weight requirements for the equipment, retransmission of lost packages could not be afforded. However, minor occurrences of package loss were not considered as a problem by the Foulum research group.

Some wireless sensor networks support a multi-hop topology in which nodes can interact with each other to transmit and receive data when out of range of a base station. SPINE, used in this project, supports only a single-hop star-topology without immediate retransmission capabilities (IEEE 802.15.4). Metal bars and biological matter were expected to reduce the effectiveness of real-time data coverage, and retransmission of lost messages was deemed too costly in terms of battery efficiency. Utilizing a multi-hop topology in such an environment will effectively turn every ear tag into a basestation, thus reducing the complexity of the base-station infrastructure. This reduction in complexity, however, increases the amount of required transmissions for an ear tag drastically, thus reducing the lifetime of most ear tags in the sensor network depending on their ability to reach the main base station. To avoid disturbance of the radio channel in this situation, a second ear tag could be mounted at the other ear. This suggestion would ensure that a radio link is available most of the time independent of sow behaviour, though leading to a higher cost of the system and possibly more redundant data.

The TinyOS and SPINE frameworks facilitated building a system for collecting biological data. Combined, the two frameworks supplied the development team features such as over-the-air configuration of multiple nodes as well as fast integration of new drivers for custom sensor extensions. Thus, using TinyOS and SPINE provided us with the required high flexibility for adapting and updating the sensor platform during the progressive iterations of the project in a much easier manner than by using a dedicated platform. The platform allowed us to deploy new versions of data gathering and communication algorithms even after the devices were deployed in the farrowing pen environment. However, the Shimmer platform did have some shortcomings. First of all, it did not allow us to pursue a local caching strategy due to the lack of low power local storage facilities. Also, it did not enable us to choose an alternative radio platform which might perform better in the pen environment. Here, a dedicated platform, such as the solution suggested by Marchioro et al. (2011), might be better suited. That said, a dedicated platform is costly to develop and maintain, is more difficult to extend with new drivers, and does not allow for an iterative progressive research agenda as required by the Foulum research group as previously discussed in this paper.

If the ear tags described in this paper are to be used commercially by farmers, it will be necessary to implement specific, behavioural algorithms in the ear tags software so that data is transmitted only if a certain pattern is observed. This would allow a sow to keep the same ear tag during its entire lifetime due to the greatly lowered frequency of radio-activation and optimized use of the efficient TI MSP340 CPU of the Shimmer platform. 


\section{Conlusion}

We found that it is feasible to construct an ear mounted flexible wireless sensor infrastructure platform that is easy to reconfigure and deploy, and able to support an iterative and progressive research agenda. Also, we found that the wireless sensor platform was sufficiently robust for real world deployment in the harsh environment of a pig pen, thus meeting the main objectives of the 15 pig farrowing studies carried out by the AU Foulum research group.

\section{References}

Cornou, C., Lundbye-Christensen, S., 2008. Classifying sows' activity types from acceleration patterns: An application of the multi-process kalman filter. Applied Animal Behaviour Science 111 (3-4), $262-273$. doi: 10.1016/j.applanim.2007.06.021

Cornou, C., Lundbye-Christensen, S., 2010. Classification of sows' activity types from acceleration patterns using univariate and multivariate models. Computers and Electronics in Agriculture 72 (2), 53 - 60. doi: $10.1016 /$ j.compag.2010.01.006

Cornou, C., Lundbye-Christensen, S., 2012. Modeling of sows diurnal activity pattern and detection of parturition using acceleration measurements. Computers and Electronics in Agriculture 80, 97-104. doi: $\underline{10.1016 / \text { j.compag.2011.11.001 }}$

Fortino, G., Guerrieri, A., Bellifemine, F., Giannantonio, R., July 2009. Spine2: developing bsn applications on heterogeneous sensor nodes. In Industrial Embedded Systems, 2009. SIES '09. IEEE International Symposium n. pp. 128-131. doi: $10.1109 /$ sies.2009.5196205

Frost, A. R., Schofield, C. P., Beaulah, S. A., Mottram, T. T., Lines, J. A., Wathes, C. M., 1997. A review of livestock monitoring and the need for integrated systems. Computers and Electronics in Agriculture 17 (2), 139 159, livestock Monitoring. doi: 10.1016/s0168-1699(96)01301-4

Giannantonio, R., Gravina, R., Kuryloski, P., Seppa, V.-P., Bellifemine, F., Hyttinen, J., Sgroi, M., April 2009. Performance analysis of an activity monitoring system using the spine framework. In: Pervasive Computing Technologies for Healthcare, 2009. PervasiveHealth 2009. 3rd International Conference on. pp. 1-8. doi: 10.4108 /icst.pervasivehealth2009.6004

Guo, Y., Poulton, G., Corke, P., Bishop-Hurley, G., Wark, T., Swain, D., 2009. Using accelerometer, high sample rate gps and magnetometer data to develop a cattle movement and behaviour model. Ecological Modelling 220 (17), 2068 - 2075. doi: 10.1016/j.ecolmodel.2009.04.047

Johnson, M., Healy, M., Van De Ven, P., Hayes, M. J., Nelson, J., Newe T., Lewis, E., 2009. A comparative review of wireless sensor network mote technologies. Christchurch, New zealand, pp. $1439-1442$. doi: $10.1109 /$ icsens.2009.5398442

Marchioro, G. F., Cornou, C., Kristensen, A. R., Madsen, J., 6 2011. Sows activity classification device using acceleration data. a resource constrained approach. Computers and Electronics in Agriculture 77 (1), $110-117$. doi: 10.1016/j.compag.2011.04.004

Moreau, M., Siebert, S., Buerkert, A., Schlecht, E., 2009. Use of a triaxial accelerometer for automated recording and classification of goats grazing behaviour. Applied Animal Behaviour Science 119 (3), 158-170. doi: 10.1016/j.applanim.2009.04.008

Oliviero, C., Pastell, M., Heinonen, M., Heikkonen, J., Valros, A., Ahokas, J., Vainio, O., Peltoniemi, O. A. T., 6 2008. Using movement sensors to detect the onset of farrowing. Biosystems Engineering 100 (2), $281-285$. doi: $10.1016 /$ j.biosystemseng.2008.03.008

Roelofs, J., Eerdenburg, F. V., Soede, N., Kemp, B., 2005. Various behavioural signs of estrous and their relationship with time of ovulation in dairy cattle. Theriogenology 63 (5), 1366-1377.

doi: 10.1016/j.theriogenology.2004.07.009

Ulrich, B., Stollberg, U., Holz, R., Schleusener, T., June 2008. Alt pedometer-new sensor-aided measurement system for improvement in oestrus detection. Comput. Electron. Agric. 62, 73-80. doi: $10.1016 /$ j.compag.2007.08.014

Wathes, C., Kristensen, H., Aerts, J.-M., Berckmans, D., 2008. Is precision livestock farming an engineer's daydream or nightmare, an animal's friend or foe, and a farmer's panacea or pitfall? Computers and Electronics in Agriculture 64 (1), 2 - 10, smart Sensors in precision livestock farming. doi: 10.1016/j.compag.2008.05.005 
Yang, A. Y., Wicker, S., Gravina, R., Shia, V., Yan, P., Giani, A., Sastry, S., Bajcsy, R., Seto, E., Kuryloski, P., Gilani, K., Giannantonio, R., Seppa, V. P., Wang, C., Hyttinen, J., June 2009. Dexternet: An open platform for heterogeneous body sensor networks and its applications. In: Wearable and Implantable Body Sensor Networks, 2009. BSN 2009. Sixth International Workshop on. pp. 92-97. doi: $\underline{10.1109 / \text { bsn.2009.31 }}$ 